\title{
Clinical Efficacy of Injection and Mechanical Therapy for Peyronie's Disease: A Systematic Review of the Literature
}

\author{
Giorgio Ivan Russo $^{a, *}$, Uros Milenkovic ${ }^{b}$, Wayne Hellstrom $^{c}$, Laurence A. Levine ${ }^{d}$, David Ralph ${ }^{e}$, \\ Maarten Albersen ${ }^{b}$ \\ ${ }^{a}$ Urology Section, Department of Surgery, University of Catania, Catania, Italy; ${ }^{\mathrm{b}}$ Department of Urology, University Hospitals Leuven, Leuven, Belgium; \\ ${ }^{\mathrm{c}}$ Department of Urology, Tulane University School of Medicine, New Orleans, LA, USA; ${ }^{\mathrm{d}}$ Department of Urology, Rush University Medical Center, Chicago, IL,

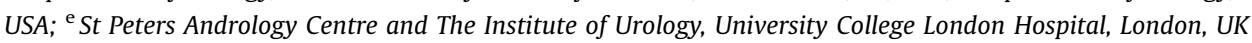

\section{Article info}

\section{Article history:}

Accepted July 7, 2018

Associate Editor:

Jean-Nicolas Cornu

\section{Keywords:}

Collagenase Clostridium

histolyticum

Hyaluronic acid

Interferon $\alpha-2 b$

Intralesional therapy

Penile curvature

Penile traction

Peyronie's disease

Vacuum device

Verapamil

\begin{abstract}
Context: Conservative therapies for Peyronie's disease (PD) aim to treat early-stage disease by improving clinical outcomes such as penile curvature, penile length erectile dysfunction, and pain.

Objective: To summarise the available evidence regarding injection and mechanical therapies for PD treatment.

Evidence acquisition: An extensive search of Medline, Embase, and Scopus databases retrieved English-language articles up to 15 May 2018. The systematic review protocol was registered on PROSPERO (CRD42017077050). Inclusion criteria were as follows: adults with early or chronic PD receiving treatment with injection therapy, penile traction, or a vacuum device.

Evidence synthesis: Fifty-two articles were selected according to the inclusion criteria17 comparative studies and 35 cohort studies-analysing the effects of calcium channel blockers (verapamil, nicardipine), collagenase Clostridium histolyticum (CCH), interferon $\alpha-2 b$ (IFN $\alpha-2 b)$, hyaluronic acid (HA), onabotulinum toxin A, thiocolchicine, penile extender devices, and vacuum devices. Qualitative data from these studies suggest a clinically significant effect of $\mathrm{CCH}$ and IFN $\alpha-2 \mathrm{~b}$ injection therapy to decrease penile curvature and conserve penile length. Verapamil and HA performed well in single-arm or case-control studies. Studies of penile traction and vacuum devices in patients with PD demonstrated some benefits in terms of curvature and penile length but only in small, underpowered, nonrandomised studies. Studies of onabotulinum toxin A injections or thiocolchicine showed significant improvements in penile curvature, but only in studies of single-arm or case-control design.

Conclusions: $\mathrm{CCH}$ and IFN $\alpha-2 \mathrm{~b}$ injected into stable PD plaques can decrease penile curvature; $\mathrm{CCH}$ can also improve penile length. However, based on available data, strong conclusions cannot be drawn for other treatments, including calcium channel blockers, HA, or mechanical therapies.

Patient summary: Our systematic review of injection therapies provides evidence for improvements in penile curvature for patients with Peyronie's disease. No robust evidence is available to support the use of other local treatments including calcium channel blockers, hyaluronic acid, or mechanical therapies.

(C) 2018 European Association of Urology. Published by Elsevier B.V. All rights reserved.

${ }^{*}$ Corresponding author. Urology Section, University of Catania, Via Santa Sofia 78, 95125, Italy. Tel. +390953782710.

E-mail address: giorgioivan1987@gmail.com (G.I. Russo).




\section{Introduction}

Peyronie's disease (PD) is a progressive fibrotic disorder of the tunica albuginea that causes penile deformity, making sexual intercourse difficult. It can result in significant psychological stress for patients and partners alike. PD occurs in $\sim 22.4-25.7$ per 100000 men, with the highest incidence in men aged 50-59 yr [1]. Current conservative therapies for PD aim to treat patients in the early stages of the disease. Several options are available, including oral pharmacotherapy, intralesional injection therapy, topical treatments and penile extender and vacuum devices [2]. The rationale for intralesional therapy is based on the inflammatory and fibrotic aetiopathology of the disease $[1,3]$.

Level 1 evidence regarding intralesional and local mechanical treatment is currently lacking, and outcomes of different studies are difficult to compare owing to heterogeneity between protocols. Injection therapies have become popular to reduce penile pain (PP), plaque size (PS), and/or penile curvature (PC). In 2013, collagenase Clostridium histolyticum $(\mathrm{CCH})$ was introduced as the first (and to date only) Food and Drug Administration- and European Medicines Agency-approved treatment option for PD [4,5]. CCH has been shown in consecutive randomised controlled trials (RCTs) to reduce PC in men with PD [6]. However, a plethora of intralesional therapies are being offered to patients, despite a lack of efficacy data. Studies of local injectables and mechanical therapies for PD have produced contradictory results, and poor study design, including lack of placebo, limited number of participants, short-term follow-up, and different outcome measures, make it difficult to draw recommendations for the use of these therapies in a real-life setting [7].

Based on these limitations, this systematic review summarises and critically interprets the available evidence regarding injection therapies, penile extenders, or vacuum pump device use for the treatment of PD.

\section{Evidence acquisition}

An extensive search of Medline, Embase, and Scopus databases was conducted to retrieve English-language articles published up to 15 May 2018 (PROSPERO registration [CRD42017077050]). Search terms are shown in the Supplementary material and the protocol was performed according to the Preferred Reporting Items for Systematic Reviews and Meta-analyses (PRISMA) statement [8].

Retrieved studies had to include adults with early or chronic PD receiving injection therapy or treatment with a penile extender or vacuum device.

Retrospective studies, prospective clinical studies with single-arm design, or comparative, placebo-controlled clinical trials were included in the qualitative analysis. Congress meetings, editorial comments, and review papers were excluded. Studies describing surgical interventions and oral therapy alone were excluded from the analysis. Other exclusion criteria were as follows: case reports, extracorporeal shock wave therapy, and full texts not available in the English language.

Two reviewers (G.I.R. and U.M.) independently assessed the eligibility of the identified papers (96\% agreement), and any disagreements were discussed with a third reviewer (M.A.).

The primary end point of this systematic review was clinical efficacy of PD therapies to reduce PC; secondary end points were changes in PS, erectile function (EF), PP, and penile length (PL). Studies were included if at least one of the previously listed end points were assessed. In case of studies that included the same patient cohort but conducted assessments at different time points, the most recent study has been included.

\section{Evidence synthesis}

\section{1. $\quad$ Literature search results}

After removing duplicates, a total of 407 studies were identified by the literature search and independently screened. Of these articles, 52 fit the inclusion criteria and were further assessed for eligibility (Fig. 1): 17 comparative studies [6,9-23] and 35 noncomparative studies [3,24-59] (Tables 1 and 2). Two studies that included investigation of oral + verapamil therapy were included, and one included interferon $\alpha-2 b$ (IFN $\alpha-2 b)$ injection versus IFN $\alpha-2 b+$ Vit. E versus oral Vit. E; however, only the study arms that investigated injection therapy as a single treatment modality were assessed [3,31]. The overall characteristics of included studies are listed in Tables 1-5.

\subsection{Risk of bias assessment}

The risk of bias in all included RCTs was evaluated according to the Cochrane Collaboration risk of bias tool [60]. For nonrandomised studies, we used the ROBINS-I tool to address the methodological quality [61].

Overall, the risk of bias of RCTs was deemed to be low (Fig. 2), whereas the risk was moderate or critical for nonrandomised studies in measurement of outcomes and selection of the reported results (Fig. 3).

\subsection{Efficacy data in comparative studies}

\subsubsection{Calcium channel antagonists: verapamil or nicardipine}

A total of nine studies evaluated the clinical efficacy of verapamil; seven were randomised $[10,13,16,17,19,21,22]$ and two were nonrandomised (Table 1) [9,20].

Only two prospective nonrandomised studies were identified.

Only one study by Rehman et al. [19] compared $10 \mathrm{mg}$ of intralesional verapamil injection with placebo (saline solution), demonstrating a reduction of PC from $37.7^{\circ}$ to $29.6^{\circ}$ for verapamil compared with a reduction from $33.6^{\circ}$ to $31.4^{\circ}$ for placebo. Furthermore, verapamil was also superior to placebo in terms of PS reduction and preservation of EF. 

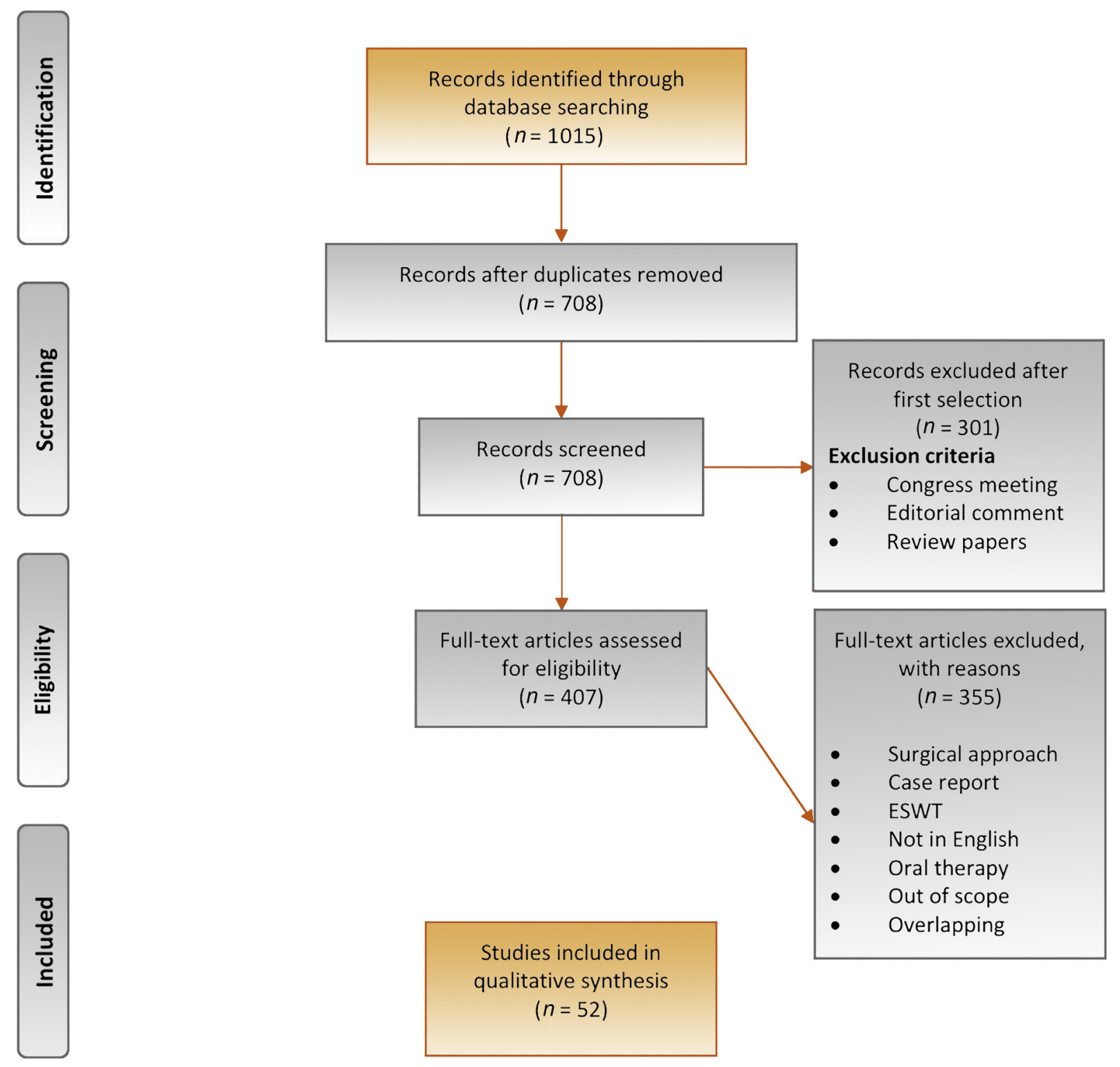

Fig. 1 - PRISMA flow diagram.

ESWT = extra corporeal shock wave therapy; PRISMA = Preferred Reporting Items for Systematic Reviews and Meta-analysis.

Soh and coworkers [21] performed a randomised placebo-controlled study to investigate the effects of $10 \mathrm{mg}$ nicardipine injections versus placebo. This study showed no effect on PC but showed an effect on PP.

Three studies used a randomised, double-blind, placebocontrolled, partial cross-over design to compare verapamil applied using transdermal electromotive administration (TEA) with placebo + TEA [13], verapamil versus verapamil + TEA [16], and verapamil + TEA versus orgotein + dexamethasone [17]. Of these, the first one was not able to show a statistical PC improvement in either group [13], whereas the second [16] did not report PC as an outcome of the study; however, PP (assessed by a visual analogue score [VAS]) decreased significantly after verapamil + TEA [16]. The third study demonstrated an improvement in PC in both the verapamil + TEA and the orgotein + dexa- methasone groups, but the study did not perform intergroup analysis [17].

Favilla and colleagues [10] compared intralesional verapamil with hyaluronic acid (HA) injection, showing a significant reduction in PC in men treated with $\mathrm{HA}\left(-4.60^{\circ}\right)$ compared with baseline and the verapamil group, in which no effect was observed. An RCT by Toscano et al. [22] compared verapamil $(5 \mathrm{mg})$ with thiocolchicine $(4 \mathrm{mg})$, showing a significant improvement in PC in both groups compared with baseline. However, no intergroup comparison was performed.

Abern et al. [9] compared verapamil + penile traction + oral pentoxifylline and L-arginine (group I) with verapamil + oral pentoxifylline and L-arginine (group II), demonstrating significant decreases in PC in both groups (group I: $44.4^{\circ}$ vs $33.4^{\circ}$; group II: $36.6^{\circ}$ vs $21.5^{\circ}$ ). However, intergroup 
Table 1 - Characteristics of selected original publications on calcium channel blockers identified by systematic literature review

\begin{tabular}{|c|c|c|c|c|c|c|c|}
\hline Study & Design & Interventions & $n$ & $\begin{array}{l}\text { Baseline penile } \\
\text { curvature }\end{array}$ & Treatment protocol & $\begin{array}{l}\text { Post-treatment } \\
\text { penile curvature }\end{array}$ & $p$ value \\
\hline \multicolumn{8}{|c|}{ Comparative studies } \\
\hline $\begin{array}{l}\text { Abern et al } \\
\text { (2012) [9] }\end{array}$ & $\begin{array}{l}\text { Prospective } \\
\text { nonrandomised }\end{array}$ & $\begin{array}{l}\text { Verapamil + penile } \\
\text { traction + oral } \\
\text { pentoxifylline and } \\
\text { L-arginine (group I) vs } \\
\text { verapamil + oral } \\
\text { pentoxifylline and } \\
\text { L-arginine (group II) }\end{array}$ & $\begin{array}{l}39 \text { (group I); } \\
35 \text { (group II) }\end{array}$ & $\begin{array}{l}44.4^{\circ} \pm 27.5^{\circ} \text { (group I) } \\
36.6^{\circ} \pm 18.5^{\circ} \text { (group II) }\end{array}$ & $\begin{array}{l}10 \mathrm{mg} \text { verapamil every other week } \\
\text { for a total of } 24 \mathrm{wk}+\text { oral } \\
\mathrm{L} \text {-arginine } 1 \mathrm{~g} \text { b.i.d. and } \\
\text { pentoxifylline } 400 \mathrm{mg} \text { t.i.d. + penile } \\
\text { traction }(2-8 \mathrm{~h} \text { daily })\end{array}$ & $\begin{array}{l}33.4^{\circ} \pm 25.3^{\circ} \text { (group I); } \\
21.5^{\circ} \pm 19.3^{\circ} \text { (group II) }\end{array}$ & $\begin{array}{l}p=0.03 \text { (group I); } \\
p<0.01 \text { (group II) }\end{array}$ \\
\hline $\begin{array}{l}\text { Favilla et al } \\
\text { (2017) [10] }\end{array}$ & Randomised & Verapamil vs HA & $\begin{array}{l}69 \text { (verapamil); } \\
63 \text { (HA) }\end{array}$ & $\begin{array}{l}90.5 \geq 30^{\circ} \text { (verapamil); } \\
95.7 \% \geq 30^{\circ}(\mathrm{HA})\end{array}$ & $\begin{array}{l}\text { Intralesional verapamil injection } \\
(10 \mathrm{mg} \text { in } 5 \mathrm{ml} \text { of normal saline }) \\
\text { weekly for } 12 \mathrm{wk} \text {; intralesional } \mathrm{HA} \\
\text { injection }(0.8 \% \text { highly purified } \\
\text { sodium salt } \mathrm{HA} 16 \mathrm{mg} / 2 \mathrm{ml}) \\
\text { weekly for } 12 \mathrm{wk}\end{array}$ & $\begin{array}{l}0.0^{\circ} \pm 0.0^{\circ} \\
(\text { verapamil); } \\
4.60^{\circ} \pm 5.63^{\circ}(\mathrm{HA})\end{array}$ & $\begin{array}{l}p<0.001(\mathrm{HA}) \\
p<0.001 \mathrm{HA} \text { vs } \\
\text { verapamil }\end{array}$ \\
\hline $\begin{array}{l}\text { Greenfield et } \\
\text { al (2007) [13] }\end{array}$ & Randomised & $\begin{array}{l}\text { EMDA verapamil } \\
\text { (group I) vs EMDA } \\
\text { placebo (group II) }\end{array}$ & $\begin{array}{l}23 \text { (group I); } \\
19 \text { (group II) }\end{array}$ & $\begin{array}{l}43.5^{\circ} \pm \mathrm{NR} \text { (group I) } \\
51.1^{\circ} \pm \mathrm{NR} \text { (group II) }\end{array}$ & $\begin{array}{l}10 \mathrm{mg} \text { verapamil in } 4 \mathrm{ml} \text { saline } \\
\text { solution + Mini-Physionizer device } \\
\text { ( } 2.4 \mathrm{~mA} \text { for } 20 \mathrm{~min} \text { twice weekly) } \\
\text { for a total of } 3 \mathrm{mo}\end{array}$ & $\begin{array}{l}34^{\circ} \pm N R \text { (group I); } \\
43^{\circ} \pm N R \text { (group II) }\end{array}$ & NS \\
\hline $\begin{array}{l}\text { Mehrsai et al } \\
\text { (2013) [16] }\end{array}$ & Randomised & $\begin{array}{l}\text { Verapamil vs TEA } \\
\text { verapamil }\end{array}$ & $\begin{array}{l}30 \text { (verapamil); } \\
30 \text { (TEA) }\end{array}$ & NR & $\begin{array}{l}6 \text { weekly doses of } 10 \mathrm{mg} \text { verapamil } \\
+4 \mathrm{mg} \text { dexamethasone diluted in } \\
2 \mathrm{ml} \text { distilled water by TEA or } \\
\text { intralesional injection }\end{array}$ & NR & - \\
\hline $\begin{array}{l}\text { Montorsi et } \\
\text { al (2000) [17] }\end{array}$ & $\begin{array}{l}\text { Randomised, } \\
\text { double-blind, } \\
\text { placebo-controlled, } \\
\text { partial cross-over } \\
\text { design (group I); } \\
\text { uncontrolled } \\
\text { (group II) }\end{array}$ & $\begin{array}{l}\text { EMDA } 8 \mathrm{mg} \text { of } \\
\text { orgotein }+8 \mathrm{mg} \text { of } \\
\text { dexamethasone } \\
+120 \mathrm{mg} \text { of lidocaine } \\
\text { (group I) vs EMDA } \\
\text { verapamil (group II) }\end{array}$ & $\begin{array}{l}40 \text { (group I); } \\
40 \text { (group II) }\end{array}$ & $\begin{array}{l}30^{\circ} \text { (range: } 20-45^{\circ}, \\
\text { group I); } 30^{\circ} \text { (range: } \\
20-45^{\circ}, \text { group II) }\end{array}$ & $\begin{array}{l}9 \text { sessions ( } 3 \text { times a week for } \\
3 \text { wk; sessions administered every } \\
\text { other day) a duration of } 20 \text { min } \\
\text { each }\end{array}$ & $\begin{array}{l}20^{\circ} \text { (range: } 0-45^{\circ}, \\
\text { group I); } 20^{\circ} \text { (range: } \\
0-45^{\circ}, \text { group II) }\end{array}$ & $\begin{array}{l}p<0.05 \text { (both groups } \\
\text { vs baseline) }\end{array}$ \\
\hline $\begin{array}{l}\text { Rehman et al } \\
\text { (1998) [19] }\end{array}$ & Randomised & Verapamil vs placebo & $\begin{array}{l}7 \text { (verapamil); } \\
7 \text { (placebo) }\end{array}$ & $\begin{array}{l}37.71^{\circ} \pm 9.3^{\circ}(\text { SEM; } \\
\text { verapamil); } \\
33.57^{\circ} \pm 9.7^{\circ}(\mathrm{SEM} ; \\
\text { placebo })\end{array}$ & $\begin{array}{l}10 \mathrm{mg} / 1 \mathrm{ml} \text { of verapamil; dosage } \\
10-27 \mathrm{mg}\end{array}$ & $\begin{array}{l}29.57^{\circ} \pm 7.3^{\circ}(\text { SEM; } \\
\text { verapamil); } \\
31.43^{\circ} \pm 8.9^{\circ} \text { (SEM; } \\
\text { placebo) }\end{array}$ & $\begin{array}{l}p<0.07 \text { (verapamil } \\
\text { vs placebo) }\end{array}$ \\
\hline $\begin{array}{l}\text { Shirazi et al } \\
\text { (2009) [20] }\end{array}$ & $\begin{array}{l}\text { Prospective } \\
\text { nonrandomised }\end{array}$ & Verapamil vs control & $\begin{array}{l}40 \text { (verapamil); } \\
40 \text { (control) }\end{array}$ & $\begin{array}{l}49.71^{\circ} \pm 9.3^{\circ} \\
\text { (verapamil); } \\
45.57^{\circ} \pm 9.7^{\circ} \text { (control) }\end{array}$ & $\begin{array}{l}10 \mathrm{mg} \text { of verapamil in } 10 \mathrm{ml} \text { of } \\
\text { distilled water twice weekly for } \\
12 \mathrm{wk}\end{array}$ & $\begin{array}{l}47.57^{\circ} \pm 7.3^{\circ} \\
\text { (verapamil); } \\
43.43^{\circ} \pm 8.9^{\circ} \text { (control) }\end{array}$ & $\begin{array}{l}p=0.91 \text { (verapamil vs } \\
\text { control) }\end{array}$ \\
\hline $\begin{array}{l}\text { Soh et al } \\
\text { (2010) [21] }\end{array}$ & Randomised & Nicardipine vs placebo & $\begin{array}{l}32 \text { (nicardipine); } \\
30 \text { (placebo) }\end{array}$ & NR & $\begin{array}{l}10 \mathrm{mg} \text { nicardipine (in } 10 \mathrm{ml} \text { of } \\
\text { distilled water) biweekly in } 10 \mathrm{wk} \\
\text { with a total of six sessions }\end{array}$ & NR & $\begin{array}{l}p=0.13 \text { nicardipine } \\
\text { vs placebo }\end{array}$ \\
\hline $\begin{array}{l}\text { Toscano et al } \\
\text { (2006) [22] }\end{array}$ & Randomised & $\begin{array}{l}\text { Thiocolchicine vs } \\
\text { verapamil }\end{array}$ & $\begin{array}{l}13 \text { (thiocolchicine); } \\
12 \text { (verapamil) }\end{array}$ & $\begin{array}{l}46.77^{\circ} \pm 16.48^{\circ} \\
\text { (thiocolchicine); } \\
50.42^{\circ} \pm 10.97^{\circ} \\
\text { (verapamil) }\end{array}$ & $\begin{array}{l}2 \mathrm{ml} \text { injection containing } 4 \mathrm{mg} \text { of } \\
\text { thiocolchicine; } 2 \mathrm{ml} \text { of a solution } \\
\text { containing } 5 \mathrm{mg} \text { of verapamil }\end{array}$ & $\begin{array}{l}36.23^{\circ} \pm 15.78^{\circ} \\
\text { (thiocolchicine); } \\
42.08^{\circ} \pm 13.08^{\circ} \\
\text { (verapamil) }\end{array}$ & $\begin{array}{l}p=0.019 \\
\text { (thiocolchicine); } \\
p=0.012 \text { (verapamil) }\end{array}$ \\
\hline
\end{tabular}




\begin{tabular}{|c|c|c|c|c|c|c|c|}
\hline Study & Design & Interventions & $n$ & $\begin{array}{l}\text { Baseline penile } \\
\text { curvature }\end{array}$ & Treatment protocol & $\begin{array}{l}\text { Post-treatment } \\
\text { penile curvature }\end{array}$ & $p$ value \\
\hline \multicolumn{8}{|c|}{ Single-arm studies } \\
\hline $\begin{array}{l}\text { Bennett et al } \\
(2007)[27]\end{array}$ & $\begin{array}{l}\text { Prospective } \\
\text { nonrandomised }\end{array}$ & Verapamil & 94 & $50^{\circ}\left( \pm 28^{\circ}\right)$ & $\begin{array}{l}6 \text { intralesional injections ( } 10 \mathrm{mg} \text { in } \\
5 \mathrm{ml} \text { saline) every } 2 \mathrm{wk}\end{array}$ & $47^{\circ}\left( \pm 35^{\circ}\right)$ & NS \\
\hline $\begin{array}{l}\text { Cavallini et al } \\
\text { (2007) [29] }\end{array}$ & $\begin{array}{l}\text { Prospective } \\
\text { nonrandomised }\end{array}$ & $\begin{array}{l}\text { Verapamil } 10 \mathrm{mg} / 4 \mathrm{ml} \\
\text { (group I); verapamil } \\
10 \mathrm{mg} / 10 \mathrm{ml} \text { (group II); } \\
\text { verapamil } 10 \mathrm{mg} / 20 \mathrm{ml} \\
\text { (group III) }\end{array}$ & $\begin{array}{l}27 \text { (group I); } \\
24 \text { (group II); } \\
26 \text { (group III) }\end{array}$ & $\begin{array}{l}28.1^{\circ}\left( \pm 13^{\circ} ; \text { group I); }\right. \\
27.2^{\circ}\left( \pm 11.1^{\circ} ; \text { group II }\right) \\
29.4^{\circ}\left( \pm 13.6^{\circ} \text {; group }\right. \\
\text { III })\end{array}$ & $\begin{array}{l}5-6 \text { injections every } 2 \mathrm{wk} \text {. for a } \\
\text { total of } 12 \text { sessions }\end{array}$ & $\begin{array}{l}23.4^{\circ} \pm 11.8^{\circ} \text { (group I); } \\
20.5^{\circ} \pm 8.9^{\circ} \text { (group II); } \\
15.8^{\circ} \pm 7.7^{\circ} \text { (group III) }\end{array}$ & NS \\
\hline $\begin{array}{l}\text { Dell'Atti } \\
\text { (2015) [31] }\end{array}$ & $\begin{array}{l}\text { Prospective } \\
\text { nonrandomised }\end{array}$ & Verapamil & 23 & $21.24^{\circ}$ (range $11-27^{\circ}$ ) & 1 injection/wk for $12 \mathrm{wk}$ & $19.8^{\circ}(\mathrm{NR})$ & NS \\
\hline $\begin{array}{l}\text { Favilla et al } \\
\text { (2013) [3] }\end{array}$ & $\begin{array}{l}\text { Prospective } \\
\text { randomised }\end{array}$ & $\begin{array}{l}\text { Verapamil } 10 \mathrm{mg} \text { in } \\
5 \mathrm{ml} \text { (group I); } \\
\text { verapamil + oral } \\
\text { antioxidants (group II) }\end{array}$ & $\begin{array}{l}52 \text { (group I); } \\
53 \text { (group II) }\end{array}$ & $\begin{array}{l}47.9^{\circ}(9.12 ; \text { group I }) \\
49^{\circ}(8.81 ; \text { group II })\end{array}$ & 1 injection/wk for $12 \mathrm{wk}$ & $\begin{array}{l}\text { Change: }-10.86^{\circ} \\
\left( \pm 9.5^{\circ} ; \text { group I); } 11.97^{\circ}\right. \\
\left( \pm 8.55^{\circ} ; \text { group II }\right)\end{array}$ & $\begin{array}{l}p<0.05 \text { (group I) } \\
p<0.05 \text { (group II) }\end{array}$ \\
\hline $\begin{array}{l}\text { Heidari et al } \\
\text { (2010) [34] }\end{array}$ & $\begin{array}{l}\text { Prospective } \\
\text { nonrandomised }\end{array}$ & Verapamil & 16 & $17.18^{\circ}\left( \pm 16.52^{\circ}\right)$ & $\begin{array}{l}\text { Verapamil } 10 \mathrm{mg} / \mathrm{cm}^{2} \text { every } 2 \mathrm{wk} \\
\text { for a total of } 6 \text { injections }\end{array}$ & $12.5^{\circ}\left( \pm 13.4^{\circ}\right)$ & $p<0.01$ \\
\hline $\begin{array}{l}\text { Levine et al } \\
(2002)[40]\end{array}$ & $\begin{array}{l}\text { Prospective } \\
\text { nonrandomised }\end{array}$ & Verapamil & 156 & NR & $\begin{array}{l}10 \mathrm{mg} \text { in } 10 \mathrm{ml} \text { saline every } 2 \mathrm{wk} \text {, } \\
\text { with a total of } 12 \text { injections }\end{array}$ & $\begin{array}{l}79 \text { patients reported } \\
\text { decreased curvature; } \\
41 \% \text { grade } 1\left(<30^{\circ}\right) \\
\text { improved; } 68 \% \text { grade } 2 \\
\text { improved; } 62 \% \text { grade } 3 \\
\text { improved }\end{array}$ & $p<0.01$ \\
\hline $\begin{array}{l}\text { Levine (1997) } \\
\text { [38] }\end{array}$ & $\begin{array}{l}\text { Prospective } \\
\text { nonrandomised }\end{array}$ & Verapamil & 38 & NR & $\begin{array}{l}10 \mathrm{mg} \text { in } 10 \mathrm{ml} \text { saline every } 2 \mathrm{wk} \text {, } \\
\text { with a total of } 12 \text { injections }\end{array}$ & $\begin{array}{l}50 \% \text { of grade } 1\left(<30^{\circ}\right) \\
\text { improved; } 57 \% \text { of grade } \\
2 \text { improved; } 46 \% \text { of } \\
\text { grade } 3 \text { improved }\end{array}$ & NR \\
\hline $\begin{array}{l}\text { Levine et al. } \\
\text { (1994) [41] }\end{array}$ & $\begin{array}{l}\text { Prospective } \\
\text { nonrandomised }\end{array}$ & Verapamil & 14 & $48^{\circ}$ (range: $10-90^{\circ}$ ) & $10 \mathrm{mg}$ verapamil in $10 \mathrm{ml}$ saline & $21^{\circ}\left(\right.$ range $\left.10-35^{\circ}\right)$ & NR \\
\hline $\begin{array}{l}\text { Moskovic } \\
\text { et al (2011) } \\
{[44]}\end{array}$ & $\begin{array}{l}\text { Prospective } \\
\text { nonrandomised }\end{array}$ & Verapamil & 131 & $40^{\circ}\left(\max .150^{\circ}\right)$ & $\begin{array}{l}10 \mathrm{mg} \text { verapamil in } 5 \mathrm{ml} \text { saline } \\
\text { intralesional injections once every } \\
2 \mathrm{wk} \text {, for a total of } 6 \text { injections }\end{array}$ & $\begin{array}{l}26 \% \text { patients improved } \\
\left.\text { (mean } 17^{\circ}[5]\right) ; 62 \% \\
\text { unchanged }\end{array}$ & NR \\
\hline $\begin{array}{l}\text { Wolff et al. } \\
\text { (2015) [55] }\end{array}$ & $\begin{array}{l}\text { Prospective } \\
\text { nonrandomised }\end{array}$ & Verapamil & 60 & $37.3^{\circ}\left( \pm 13.3^{\circ}\right)$ & $\begin{array}{l}6 \text { verapamil injections } 15 \mathrm{mg} \text { in } \\
6 \mathrm{ml} \text { saline, once every } 3 \mathrm{wk}\end{array}$ & $21^{\circ}\left( \pm 13^{\circ}\right)$ & $p<0.05$ \\
\hline
\end{tabular}


Table 2 - Characteristics of selected original publications on IFN-2b identified by systematic literature review

\begin{tabular}{|c|c|c|c|c|c|c|c|}
\hline Study & Design & Interventions & $n$ & Baseline penile curvature & Treatment protocol & $\begin{array}{l}\text { Post-treatment } \\
\text { penile curvature }\end{array}$ & $p$ value \\
\hline \multicolumn{8}{|l|}{ Comparative studies } \\
\hline $\begin{array}{l}\text { Hellstrom et al } \\
\text { (2006) [14] }\end{array}$ & Randomised & IFN $\alpha-2 b$ vs placebo & 53 (IFN $\alpha-2 b) ; 50$ (placebo) & $\begin{array}{l}49.9^{\circ} \pm 2.4^{\circ}(\mathrm{IFN} \alpha-2 \mathrm{~b}) \\
50.9^{\circ} \pm 2.5^{\circ} \text { (Placebo) }\end{array}$ & $\begin{array}{l}\text { Intralesional injections } \\
\text { of } 5 \times 10^{6} \mathrm{IU} \text { IFN } \alpha-2 \mathrm{~b} \\
\text { in } 10 \mathrm{ml} \text { saline weekly } \\
\text { for a total of six } \\
\text { injections }\end{array}$ & $\begin{array}{l}36.4^{\circ} \pm 2.1^{\circ}(\text { IFN } \alpha-2 b) \\
46.4 \pm 2.2^{\circ} \text { (placebo) }\end{array}$ & $\begin{array}{l}p<0.001(\text { IFN } \alpha-2 b) \\
p<0.01 \text { IFN } \alpha-2 b \text { vs } \\
\text { placebo }\end{array}$ \\
\hline $\begin{array}{l}\text { Kendirci et al (2005) } \\
\text { [15] }\end{array}$ & Randomised & IFN $\alpha-2 \mathrm{~b}$ vs placebo & 19 (IFN $\alpha-2 b) ; 20$ (placebo) & $\begin{array}{l}48.75^{\circ} \pm 4.41^{\circ}(\text { IFN } \alpha-2 b) \\
47.75^{\circ} \pm 2.84^{\circ} \text { (Placebo) }\end{array}$ & $\begin{array}{l}\text { Intralesional injections } \\
\text { of } 5 \times 10^{6} \mathrm{IU} \text { IFN } \alpha-2 \mathrm{~b} \\
\text { in } 10 \mathrm{ml} \text { saline weekly } \\
\text { for a total of six } \\
\text { injections }\end{array}$ & $\begin{array}{l}36.75^{\circ} \pm 4.53^{\circ} \\
(\text { IFN } \alpha-2 \mathrm{~b}) \\
44.10 \pm 3.02^{\circ} \\
\text { (placebo) }\end{array}$ & $\begin{array}{l}p<0.001(\text { IFN } \alpha-2 b) \\
p<0.01 \text { IFN } \alpha-2 \mathrm{~b} \text { vs } \\
\text { placebo }\end{array}$ \\
\hline Inal et al (2006) [63] & Prospective & $\begin{array}{l}\text { IFN } \alpha-2 b \text { vs oral Vit. E } \\
\text { vs IFN } \alpha-2 b+\text { oral Vit. E }\end{array}$ & NR & $33^{\circ} \pm 10.3^{\circ}$ & $5.0 \times 10^{6}$ U per $12 \mathrm{wk}$ & $31.5^{\circ} \pm 9.7^{\circ}$ & $p>0.05$ \\
\hline Yafi et al (2015) [23] & $\begin{array}{l}\text { Retrospective } \\
\text { comparative }\end{array}$ & $\begin{array}{l}\text { IFN } \alpha-2 b+\text { penile } \\
\text { traction (group I); } \\
\text { IFN } \alpha-2 b \text { (group II) }\end{array}$ & 112 & $\begin{array}{l}42.3^{\circ} \text { (SD 20.8; group I); } \\
42.2^{\circ} \text { (SD 14.6; group II) }\end{array}$ & $\begin{array}{l}\text { Biweekly injections of } \\
2 \times 10^{6} \text { IU IFN } \alpha-2 \mathrm{~b} \\
+>2 \text { h traction daily }\end{array}$ & $\begin{array}{l}\text { Change of }-8.1^{\circ} \\
\text { (SD 16; group I); }-9.9^{\circ} \\
\text { (SD 11.8; group II) }\end{array}$ & NS \\
\hline \multicolumn{8}{|l|}{ Single-arm studies } \\
\hline $\begin{array}{l}\text { Ahuja et al (1999) } \\
\text { [24] }\end{array}$ & $\begin{array}{l}\text { Prospective } \\
\text { nonrandomised }\end{array}$ & IFN $\alpha-2 b$ & 21 & NR & 1 injection $0.1 \mathrm{ml}$ & $\begin{array}{l}\text { 37.2\% improvement } \\
( \pm 30.65 \%)\end{array}$ & NR \\
\hline $\begin{array}{l}\text { Astorga et al (2000) } \\
{[26]}\end{array}$ & $\begin{array}{l}\text { Prospective } \\
\text { nonrandomised }\end{array}$ & IFN $\alpha-2 b$ & 34 & NR & $\begin{array}{l}10 \times 10^{6} \mathrm{IU} \text { in } 10 \mathrm{ml} \\
\text { saline twice weekly for } \\
14 \mathrm{wk}\end{array}$ & NR & - \\
\hline $\begin{array}{l}\text { Brake et al (2001) } \\
{[28]}\end{array}$ & $\begin{array}{l}\text { Prospective } \\
\text { nonrandomised }\end{array}$ & IFN $\alpha-2 b$ & 23 & $32^{\circ}\left(\right.$ range $\left.20-50^{\circ}\right)$ & $\begin{array}{l}3 \text { times a week for } 3 \mathrm{wk} \\
\left(2 \times 10^{6} \mathrm{IU}\right)\end{array}$ & $\begin{array}{l}\text { Only improved in } \\
1 \text { patient }\end{array}$ & NR \\
\hline $\begin{array}{l}\text { Dang et al. (2004) } \\
\text { [30] }\end{array}$ & $\begin{array}{l}\text { Prospective } \\
\text { nonrandomised }\end{array}$ & IFN $\alpha-2 b$ & 21 & NR & $\begin{array}{l}\text { Biweekly injections } \\
\left(2 \times 10^{6} \mathrm{IU}\right) \text { for } 6 \mathrm{wk}\end{array}$ & $\begin{array}{l}14 \text { patients improved } \\
>20 \%\end{array}$ & NR \\
\hline $\begin{array}{l}\text { Judge and } \\
\text { Wisniewski (1997) } \\
{[36]}\end{array}$ & $\begin{array}{l}\text { Prospective } \\
\text { nonrandomised }\end{array}$ & IFN $\alpha-2 b$ & 13 & $45^{\circ}\left(\right.$ range $\left.30-75^{\circ}\right)$ & $\begin{array}{l}3 \text { times a week for } 3 \mathrm{wk} \\
\left(1.5 \times 10^{6} \mathrm{IU}\right)\end{array}$ & $12^{\circ}(\mathrm{NR})$ & NR \\
\hline Polat et al (1997) [47] & $\begin{array}{l}\text { Prospective } \\
\text { nonrandomised }\end{array}$ & IFN $\alpha-2 b$ & 15 & $\begin{array}{l}10 \text { patients }<30^{\circ} \text {; } \\
5 \text { patients }>30^{\circ}\end{array}$ & $\begin{array}{l}3 \text { times a week for } 2-4 \\
\text { wk }\left(3 \times 10^{6} \mathrm{IU}\right)\end{array}$ & $\begin{array}{l}10 \text { patients with }<30^{\circ} \\
\text { recovered completely; } \\
\text { all other patients } \\
\text { improved } 10-20^{\circ}\end{array}$ & NS \\
\hline $\begin{array}{l}\text { Stewart et al (2015) } \\
\text { [51] }\end{array}$ & Retrospective & IFN $\alpha-2 b$ & 131 & NR & $\begin{array}{l}\text { Biweekly injection } \\
\left(2 \times 10^{6} \mathrm{IU}\right) \text { for } 6 \mathrm{wk}\end{array}$ & $34.1^{\circ}\left( \pm 21.6^{\circ}\right)$ & NR \\
\hline $\begin{array}{l}\text { Wegner et al (1995) } \\
\text { [53] }\end{array}$ & $\begin{array}{l}\text { Prospective } \\
\text { nonrandomised }\end{array}$ & IFN $\alpha-2 b$ & 25 & $16.6^{\circ}$ (range $7-65^{\circ}$ ) & $\begin{array}{l}1 \times 10^{6} \text { IU of IFN } \alpha-2 b \\
\text { weekly for } 5 \mathrm{wk}\end{array}$ & NR & - \\
\hline $\begin{array}{l}\text { Wegner et al (1997) } \\
{[54]}\end{array}$ & $\begin{array}{l}\text { Prospective } \\
\text { nonrandomised }\end{array}$ & IFN $\alpha-2 b$ & 30 & $10^{\circ}\left(\right.$ range $\left.0-45^{\circ}\right)$ & $\begin{array}{l}3 \text { injections of } \\
3 \times 10^{6} \mathrm{IU} \text { of IFN } \alpha-2 b\end{array}$ & $\begin{array}{l}\text { Improvement in } \\
1 \text { patient }\end{array}$ & - \\
\hline
\end{tabular}




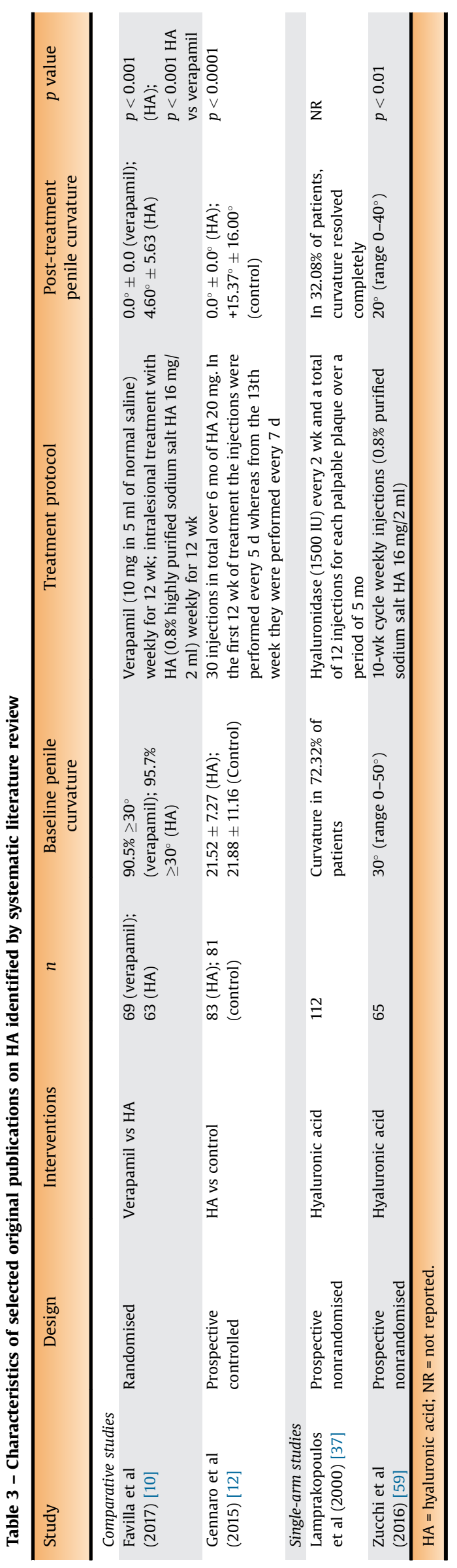

analysis was not performed. Shirazi et al. [20] performed a comparative study with a control group showing no benefit of verapamil injections on any of the end points.

Based on these data, the evidence is not robust enough to support the use of verapamil injections and does not suggest a meaningful improvement in PC compared with placebo. In fact, most of the studies investigating verapamil therapy did not perform direct statistical comparison between groups, leading to a high risk of bias.

\subsubsection{Interferon $\alpha-2 b$}

Three studies analysed clinical efficacy of IFN $\alpha-2 b$, two randomised versus placebo $[14,15]$ and one retrospective study [23] (Table 2). In their randomised controlled study, Kendirci et al. [15] demonstrated superiority of IFN $\alpha-2 b$ versus placebo for PC ( $36.8^{\circ}$ vs $\left.44.1^{\circ}\right)$ but not for PS ( 3.45 vs $3.9 \mathrm{~cm}^{2}$ ). Furthermore, Hellstrom and colleagues [14] demonstrated a clinical benefit of IFN $\alpha$-2b compared with placebo for PC ( $36.4^{\circ}$ vs $\left.46.4^{\circ}\right)$ and PS (2.2 vs $\left.3.6 \mathrm{~cm}^{2}\right)$, but not for EF or PP.

A retrospective study by Yafi et al. [23] compared IFN $\alpha$ $2 b+$ penile traction (group I) with IFN $\alpha-2 b$ alone (group II). However, the authors did not demonstrate clinical benefit compared with baseline in either group in terms of PC or PL.

These data suggest that injection therapy with IFN $\alpha-2 b$ improves PC, based on two randomised, placebo-controlled trials. However, the addition of traction failed to provide additional clinical benefit in terms of either PC or PL.

\subsubsection{Hyaluronic acid}

Gennaro et al. [12] compared injection of $20 \mathrm{mg}$ HA with a control group of patients who received no treatment, showing that HA therapy stabilised PC at a 12-mo follow-up period after HA treatment $\left(+0.0^{\circ}\right)$, compared with the control group $\left(+15 \cdot 4^{\circ}\right)$. HA injections also reduced PS $(-0.73$ $\mathrm{mm}^{3}$ ) and improved $\mathrm{EF}$, according to the International Index of Erectile Function questionnaire 15 (IIEF-15; +1.24), compared with the control group. No differences were observed in the PP VAS ( -1.66 HA vs -1.5 control; Table 3 ).

The efficacy of HA according to this non-placebocontrolled study seems to be limited, as no improvement in PC was reported. Moreover, the slight improvements in PS and EF do not translate into clinically significant benefits.

\subsection{4. $\quad \mathrm{CCH}$}

Two randomised prospective studies have compared $\mathrm{CCH}$ therapy against placebo [6,11]: one RCT compared $\mathrm{CCH}$ + vacuum + modelling with $\mathrm{CCH}+$ vacuum [18] and one prospective nonrandomised study compared $\mathrm{CCH}+$ penile traction with $\mathrm{CCH}$ alone [58] (Table 4). Gelbard and colleagues [11] first reported a subjective positive response to $\mathrm{CCH}$ therapy, demonstrating $36 \%$ and $4 \%$ improvements in PC after $\mathrm{CCH}$ and placebo, respectively.

In 2013, results from the IMPRESS I and II RCT demonstrated that $\mathrm{CCH}$ was superior to placebo in terms of percentage improvement in PC $(-34 \%$ and $-18.2 \%$, respectively), EF (mean change in IIEF- 15 of +1.0 and +0.4 , respectively), and $\mathrm{PL}(0.4$ and $0.2 \mathrm{~cm}$, respectively), but not when considering PP [6]. 
Table 4 - Characteristics of selected original publications on CCH identified by systematic literature review

\begin{tabular}{|c|c|c|c|c|c|c|c|}
\hline Study & Design & Interventions & $n$ & Baseline penile curvature & Treatment protocol & $\begin{array}{l}\text { Post-treatment } \\
\text { penile curvature }\end{array}$ & $p$ value \\
\hline \multicolumn{8}{|c|}{ Comparative studies } \\
\hline $\begin{array}{l}\text { Gelbard et al } \\
\text { (1993) [11] }\end{array}$ & Randomised & $\begin{array}{l}\text { Collagenase vs } \\
\text { placebo }\end{array}$ & $\begin{array}{l}22(\mathrm{CCH}) ; 27 \\
\text { (placebo) }\end{array}$ & $\begin{array}{l}\text { Category } 1 \text { patients were } \\
\text { characterised as those with } \\
\text { a bend of } \leq 30^{\circ} \text {, and/or } \\
\text { palpable plaque }<2 \mathrm{~cm} \text { in } \\
\text { extent. Category } 2 \text { patients } \\
\text { displayed } 30-60^{\circ} \text { angular } \\
\text { deformity and } / \text { or } 2-4 \mathrm{~cm} \text { of } \\
\text { palpable plaque in } \\
\text { maximal dimension. } \\
\text { Category } 3 \text { patients were } \\
\text { those with }>60^{\circ} \text { angular } \\
\text { deviation and/or }>4 \mathrm{~cm} \text { of } \\
\text { palpable plaque }\end{array}$ & $\begin{array}{l}6000 \text { units for category } \\
1,10000 \text { units for } \\
\text { category } 2 \text {, and } \\
14000 \text { units for } \\
\text { category } 3 \text { patients }\end{array}$ & $\begin{array}{l}\text { Subjective positive } \\
\text { response. Overall: } 36 \% \\
\text { (CCH) and 4\% } \\
\text { (placebo). Category 1: } \\
100 \%(\mathrm{CCH} \text { ) and } 25 \% \\
\text { (placebo). Category 2: } \\
36 \% \text { (CCH) and 0\% } \\
\text { (placebo). Category 3: } \\
13 \%(\mathrm{CCH}) \text { and } 0 \% \\
\text { (placebo) }\end{array}$ & $\begin{array}{l}p<0.007 \text { (overall); } \\
p=0.03 \text { (category 2) }\end{array}$ \\
\hline $\begin{array}{l}\text { Gelbard et al } \\
\text { (2013) [6] }\end{array}$ & Randomised & $\begin{array}{l}\text { Collagenase vs } \\
\text { placebo }\end{array}$ & $\begin{array}{l}401(\mathrm{CCH}) ; 211 \\
\text { (placebo) }\end{array}$ & $\begin{array}{l}50.1^{\circ} \pm 14.4^{\circ}(\mathrm{CCH}) \\
49.3^{\circ} \pm 14.0^{\circ} \text { (placebo) }\end{array}$ & $\begin{array}{l}3 \text { cycles of } 2 \text { injections } \\
\text { of CCH }(0.58 \mathrm{mg}) \text { with } \\
\text { an interval of } \sim 24-72 \mathrm{~h} \\
\text { between each injection }\end{array}$ & $\begin{array}{l}33.1^{\circ} \pm 16.8^{\circ}(\mathrm{CCH}) \\
40.0^{\circ} \pm 16.2^{\circ} \text { (placebo) }\end{array}$ & $p<0.0001$ \\
\hline $\begin{array}{l}\text { Ralph et al. } \\
\text { (2017) [18] }\end{array}$ & Randomised & $\begin{array}{l}\text { Collagenase } \\
\text { + vacuum } \\
\text { + modelling (group } \\
\text { I) vs. collagenase } \\
\text { + vacuum (group II) }\end{array}$ & $\begin{array}{l}15 \text { (group I); } 15 \\
\text { (group II) }\end{array}$ & $\begin{array}{l}59.0 \pm 15.0(\text { group I) } \\
58.3 \pm 12.2(\text { group II) }\end{array}$ & $\begin{array}{l}\text { Two intralesional } \\
\text { injections of } \mathrm{CCH} \\
0.58 \mathrm{mg} \text { separated by } \\
\sim 24-72 \mathrm{~h} \text { for four } \\
\text { cycles } \sim 6 \mathrm{wk} \text { long } \\
+ \text { vacuum twice daily } \\
\text { (morning and evening) } \\
\text { from } 14 \pm 2 \mathrm{~d} \text { after the } \\
\text { second injection of } \\
\text { CCH in each treatment } \\
\text { cycle until the start of } \\
\text { the next cycle }\end{array}$ & $\begin{array}{l}\text { Change of - } \\
23.7^{\circ} \pm 10.9^{\circ} \text { (group I); } \\
\text { change of- } 23.3^{\circ} \pm 7.2^{\circ} \\
\text { (group II) }\end{array}$ & NS \\
\hline $\begin{array}{l}\text { Ziegelmann } \\
\text { et al (2017) } \\
\text { [62] }\end{array}$ & $\begin{array}{l}\text { Prospective } \\
\text { nonrandomised study }\end{array}$ & $\begin{array}{l}\mathrm{CCH}+\text { penile } \\
\text { traction (group I) } \\
\text { vs } \mathrm{CCH} \text { (group II) }\end{array}$ & $\begin{array}{l}35 \text { (group I); } 16 \\
\text { (group II) }\end{array}$ & $\begin{array}{l}55.7^{\circ}\left( \pm 19.3^{\circ} ; \text { group I); }\right. \\
50.8^{\circ}\left( \pm 19.8^{\circ} ; \text { group II }\right)\end{array}$ & $\begin{array}{l}2 \mathrm{CCH} \text { injections per } \\
\text { series with } 4 \text { total } \\
\text { series recommended } \\
+ \text { modelling with a } \\
\text { flaccid penis for a } \\
\text { minimum of } 3 \mathrm{~h} \text { daily }\end{array}$ & $\begin{array}{l}16.1^{\circ}\left( \pm 14.9^{\circ} ; \text { group I }\right) \\
20.8^{\circ}\left( \pm 15.3^{\circ} ; \text { group II }\right)\end{array}$ & $\begin{array}{l}p<0.001 \text { (group I) } \\
p<0.001 \text { (group II) }\end{array}$ \\
\hline \multicolumn{8}{|c|}{ Single-arm studies } \\
\hline $\begin{array}{l}\text { Anaissie et al. } \\
\text { (2017) [25] }\end{array}$ & Retrospective & $\mathrm{CCH}$ & 77 & $58.2^{\circ}\left( \pm 17.9^{\circ}\right)$ & $\begin{array}{l}3 \text { cycles of } 2 \text { injections } \\
\text { of CCH }(0.58 \mathrm{mg}) \text { with } \\
\text { an interval of } \sim 24-72 \mathrm{~h} \\
\text { between each injection }\end{array}$ & $41^{\circ}\left( \pm 17^{\circ}\right)$ & NR \\
\hline $\begin{array}{l}\text { Cocci et al } \\
\text { (2018) [64] }\end{array}$ & Prospective & $\mathrm{CCH}$ & 15 & 135 & $\begin{array}{l}3 \text { intralesional } \\
\text { injections of } 0.9 \mathrm{mg} \text { of } \\
\mathrm{CCH} \text { performed at } 4- \\
\text { wk intervals at the } \\
\text { point of maximum } \\
\text { curvature }\end{array}$ & $\begin{array}{l}-19.07^{\circ}\left(-30.0^{\circ} ;-\right. \\
\left.15.0^{\circ}\right)\end{array}$ & $p<0.01$ \\
\hline
\end{tabular}




\begin{tabular}{|c|c|c|c|c|c|c|c|}
\hline Study & Design & Interventions & $n$ & Baseline penile curvature & Treatment protocol & $\begin{array}{l}\text { Post-treatment } \\
\text { penile curvature }\end{array}$ & $p$ value \\
\hline $\begin{array}{l}\text { Goldstein } \\
\text { et al (2017) } \\
\text { [32] }\end{array}$ & $\begin{array}{l}\text { Prospective } \\
\text { nonrandomised }\end{array}$ & $\mathrm{CCH}$ & 189 & $46.9^{\circ}\left( \pm 12^{\circ}\right)$ & $\begin{array}{l}\text { Maximum } 4 \text { cycles of } \\
2 \text { injections of } \mathrm{CCH} \\
(0.58 \mathrm{mg}) \text { with an } \\
\text { interval of } \sim 24-72 \mathrm{~h} \\
\text { between each injection }\end{array}$ & $\begin{array}{l}\text { Change }-36.3 \% \\
( \pm 30.72 \%)\end{array}$ & $p<0.01$ \\
\hline $\begin{array}{l}\text { Jordan (2008) } \\
{[35]}\end{array}$ & $\begin{array}{l}\text { Prospective } \\
\text { nonrandomised }\end{array}$ & $\mathrm{CCH}$ & 25 & $55.8^{\circ}\left(\right.$ range $\left.15-75^{\circ}\right)$ & $\begin{array}{l}3 \text { injections } 10000 \mathrm{IU} / \\
0.25 \mathrm{~cm}^{3} \text { per injection } \\
\text { administered over 7- } \\
10 \mathrm{~d}\end{array}$ & Change $-12.7^{\circ}$ (NR) & $p<0.05$ \\
\hline $\begin{array}{l}\text { Levine et al } \\
\text { (2015) [39] }\end{array}$ & $\begin{array}{l}\text { Prospective } \\
\text { nonrandomised }\end{array}$ & $\mathrm{CCH}$ & 347 & $53^{\circ}(14.82)$ & $\begin{array}{l}\text { Maximum } 4 \text { cycles } \\
\text { (each } 6 \text { wk) of } \\
2 \text { injections of } \mathrm{CCH} \\
(0.58 \mathrm{mg} \text { ) with an } \\
\text { interval of } \sim 24-72 \mathrm{~h} \\
\text { between each injection }\end{array}$ & $34.7^{\circ}\left( \pm 15.36^{\circ}\right)$ & $p<0.05$ \\
\hline $\begin{array}{l}\text { Nguyen et al } \\
\text { (2017) [46] }\end{array}$ & $\begin{array}{l}\text { Prospective } \\
\text { nonrandomised }\end{array}$ & $\mathrm{CCH}$ & $\begin{array}{l}\text { Acute phase } 36 \\
\text { (group I); } \\
\text { chronic phase } \\
126 \text { (group II) }\end{array}$ & $\begin{array}{l}60.6^{\circ}\left( \pm 18.5^{\circ} ; \text { group I); }\right. \\
56.9^{\circ}\left( \pm 15.2^{\circ} ; \text { group II }\right)\end{array}$ & $\begin{array}{l}\text { Maximum } 4 \text { cycles } \\
\text { (each } 6 \text { wk) of } \\
2 \text { injections of } \mathrm{CCH} \\
(0.58 \mathrm{mg} \text { ) with an } \\
\text { interval of } \sim 24-72 \mathrm{~h} \\
\text { between each injection }\end{array}$ & $\begin{array}{l}43.9^{\circ}\left( \pm 16^{\circ} ; \text { group I); }\right. \\
41.3^{\circ}\left( \pm 15.3^{\circ} ; \text { group II }\right)\end{array}$ & NS \\
\hline $\begin{array}{l}\text { Raheem et al } \\
\text { (2017) [48] }\end{array}$ & $\begin{array}{l}\text { Prospective } \\
\text { nonrandomised }\end{array}$ & $\mathrm{CCH}+$ vacuum & 53 & $54.17^{\circ}\left( \pm 15.38^{\circ}\right)$ & $\begin{array}{l}3 \text { cycles of } 1 \text { injection } \\
(0.9 \mathrm{mg})+\text { vacuum }\end{array}$ & $37^{\circ}$ (range: $12-57^{\circ}$ ) & $p<0.01$ \\
\hline $\begin{array}{l}\text { Yang et al } \\
\text { (2016) [56] }\end{array}$ & $\begin{array}{l}\text { Prospective } \\
\text { nonrandomised }\end{array}$ & $\mathrm{CCH}$ & 49 & $49.3^{\circ}$ (range: $30-100^{\circ}$ ) & $\begin{array}{l}\text { Maximum } 4 \text { cycles } \\
\text { (each } 6 \text { wk) of } \\
2 \text { injections of } \mathrm{CCH} \\
(0.58 \mathrm{mg} \text { ) with an } \\
\text { interval of } \sim 24-72 \mathrm{~h} \\
\text { between each injection }\end{array}$ & $\begin{array}{l}\text { Change }-15.4^{\circ} \text { (range: } \\
-10^{\circ} \text { to }-60^{\circ} \text { ) }\end{array}$ & $p<0.01$ \\
\hline $\begin{array}{l}\text { Ziegelmann } \\
\text { et al (2016) } \\
\text { [57] }\end{array}$ & $\begin{array}{l}\text { Prospective } \\
\text { nonrandomised }\end{array}$ & $\mathrm{CCH}$ & 27 & $60^{\circ}(20.1)$ & $\begin{array}{l}\text { Maximum } 4 \text { cycles } \\
\text { (each } 6 \text { wk) of } \\
2 \text { injections of } \mathrm{CCH} \\
(0.58 \mathrm{mg} \text { ) with an } \\
\text { interval of } \sim 24-72 \mathrm{~h} \\
\text { between each injection }\end{array}$ & $37.4^{\circ}\left( \pm 21.2^{\circ}\right)$ & $p<0.01$ \\
\hline $\begin{array}{l}\text { Ziegelmann } \\
\text { et al (2017) } \\
{[58]}\end{array}$ & $\begin{array}{l}\text { Prospective } \\
\text { nonrandomised }\end{array}$ & $\mathrm{CCH}$ & 52 & $\begin{array}{l}45^{\circ} \text { (range } 35-70^{\circ} ; \text { patient } \\
\text { perceived) }\end{array}$ & $\begin{array}{l}\text { Maximum } 4 \text { cycles } \\
\text { (each } 6 \text { wk) of } \\
2 \text { injections of } \mathrm{CCH} \\
(0.58 \mathrm{mg} \text { ) with an } \\
\text { interval of } \sim 24-72 \mathrm{~h} \\
\text { between each injection }\end{array}$ & $\begin{array}{l}\text { Change }-15^{\circ} \text { (range: }- \\
5^{\circ} \text { to }-30^{\circ} \text { ) }\end{array}$ & $p<0.01$ \\
\hline
\end{tabular}


A study by Ralph et al. [18] compared CCH + vacuum + manual modelling with $\mathrm{CCH}+$ vacuum therapy, but failed to show a significant difference between these two approaches in terms of PC reduction, PP, or EF. Similarly, a prospective nonrandomised study by Ziegelmann et al. [62] compared $\mathrm{CCH}+$ penile traction with $\mathrm{CCH}$ alone. While the PC improved from baseline by $32.6 \%$ and $32.5 \%$ respectively, there was no significant difference in PC and stretched PL between both treatment groups.

In the IMPRESS I and II clinical trials, men treated with $\mathrm{CCH}$ demonstrated a significantly greater percentage reduction in PC $(-34 \%)$ and improvement in patientreported PD bother compared with those who received placebo $(-2.8 \pm 3.8$ vs $-1.8 \pm 3.5)$. However, they also observed an $18 \%$ improvement from baseline in the placebo arm, presumably because of penile modelling. These findings raise questions regarding the putative role of penile modelling compared with placebo in improving outcomes in men with PD. Certainly, modelling seems to have an effect that is greater than the placebo effect observed in most randomised, placebo-controlled clinical trials. Further studies of $\mathrm{CCH}$ with or without penile modelling would be useful to better understand the roles of both these treatment modalities in improving PC.

\subsection{Efficacy data of single-arm studies}

3.4.1. Verapamil

A total of 12 studies have evaluated the clinical effects of verapamil using a single-arm study design $[3,27,29,31,34,38,40,41,44,50,52,55]$ (Table 1). Five studies $[3,34,40,52,55]$ demonstrated significant improvements in PC after 10 or $15 \mathrm{mg}$ intralesional verapamil, although the protocols varied widely, ranging from six to 12 injections per week. Two articles showed no statistically significant benefit of verapamil injections on the primary end point of PC $[27,31]$, nor was there a difference between groups where the authors dissolved $10 \mathrm{mg}$ of verapamil into different volumes of saline $(4,10$, and $20 \mathrm{ml})$ [29]. Three articles showed clinical improvements in PC after verapamil injections, but statistical significance was not reported, and so the evidence does not support the use of verapamil injections in PD [38,41,44].

Three studies demonstrated that verapamil $10 \mathrm{mg}$ was beneficial in terms of the secondary end points of PS reduction [3], one of which also demonstrated improvements in EF [34] and one in PP [55]. Tuygun et al. [52] evaluated the efficacy of verapamil application using electromotive drug administration (EMDA) and showed a $10^{\circ}$ reduction in PC after 2020 -min sessions at 3-d intervals over 2 mo. Finally, Riedl and colleagues [50] also evaluated verapamil $5 \mathrm{mg}$ injection with EMDA, and showed a 16\% improvement from baseline. However, the study was of poor quality, as statistical analysis was not performed.

Published data regarding injection therapy with verapamil show considerable heterogeneity, owing to the use of different dosage schedules. Moreover, the lack of placebo groups underpowers the studies, limiting their capability to provide strong efficacy data, either with or without the addition of EMDA. 
A

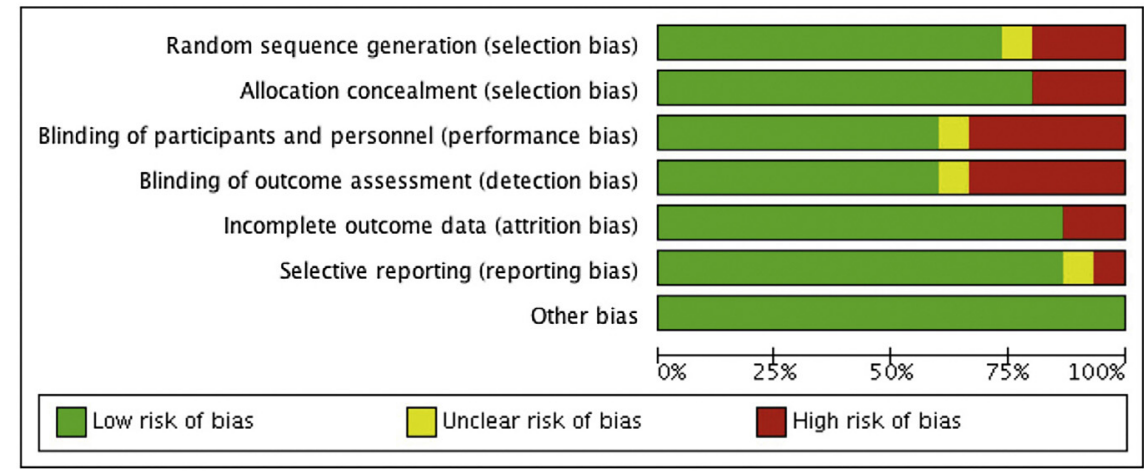

\section{B}

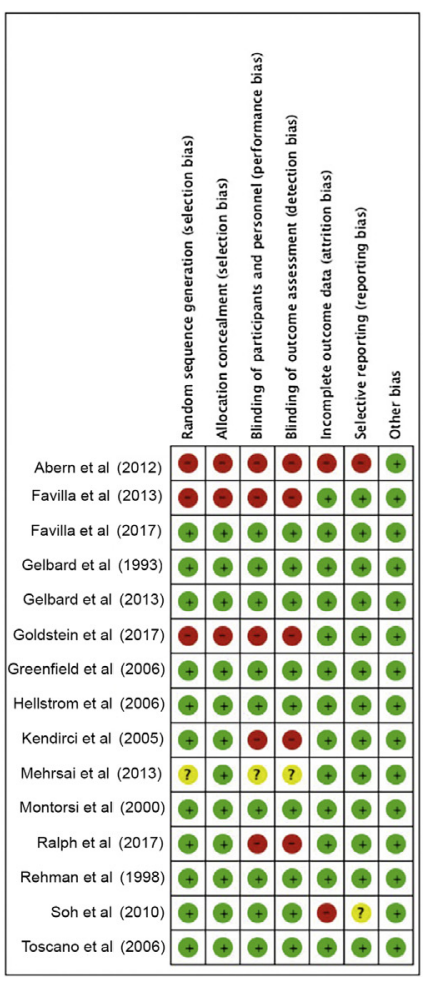

Fig. 2 - (A) Risk of bias assessment in randomised studies and (B) summary in randomised studies: review authors' judgements about each risk of bias item for each included study.

Bias due to confounding

Bias in selection of participants into the study

Bias in classification of intervention

Bias due to deviations from intended interventions

Bias due to missing data
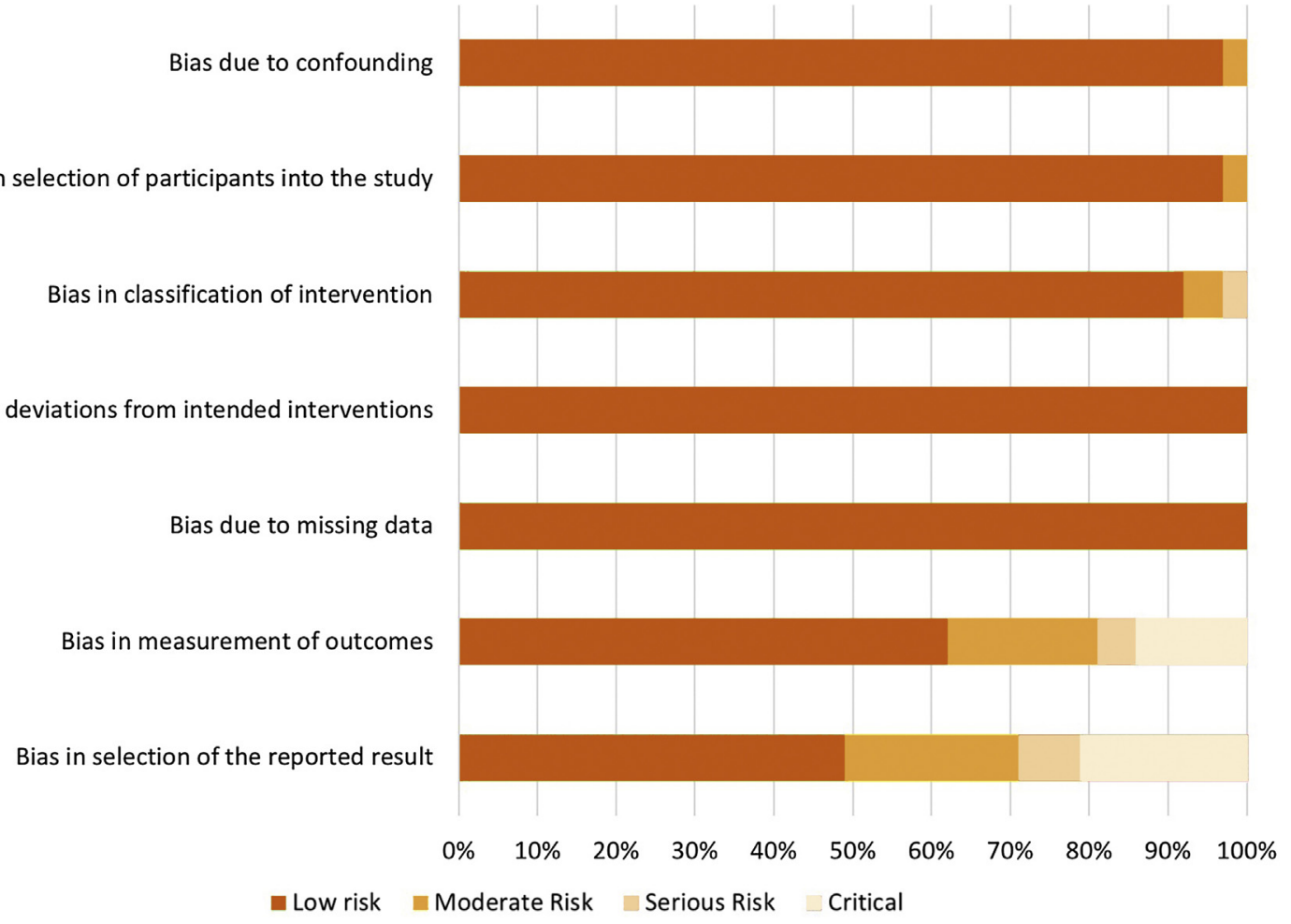

Fig. 3 - Risk of bias assessment in nonrandomised studies according to ROBINS-I tool.

ROBINS-I = risk of bias in nonrandomised studies of interventions.

Please cite this article in press as: Russo GI, et al. Clinical Efficacy of Injection and Mechanical Therapy for Peyronie's Disease: A Systematic Review of the Literature. Eur Urol (2018), https://doi.org/10.1016/j.eururo.2018.07.005 


\subsubsection{Interferon $\alpha-2 b$}

IFN $\alpha-2 b$ has been investigated in 10 separate single-arm prospective studies [24,26,28,30,36,47,51,53,54] (Table 2). However, the used treatment programmes varied widely across these studies, with doses ranging from 1 million international units (IU) to 10 million IU of IFN $\alpha-2 b$, and protocols ranging from 1 injection per week to 3 injections per week for a maximum of $14 \mathrm{wk}$. Only two studies presented characteristics of PC from baseline to final followup [36]. Judge and Wisniewski [36] reported an improvement in PC from $45^{\circ}$ at baseline to $12^{\circ}$ after IFN $\alpha-2 b$, whereas Polat et al. [47] supported the finding that IFN $\alpha-2 b$ is more efficacious in smaller plaques. There was $100 \%$ recovery of the $\mathrm{PC}$ in patients with $<30^{\circ}$ of $\mathrm{PC}$ compared with baseline. Conversely, Inal et al. [63] could not confirm these results; there was no significant improvement in PC.

Secondary outcomes of plaque reduction, pain, and EF were reported in two studies [26]. Only the report of Astorga and colleagues [26] showed a significant reduction in PS from 56.7 to $12.7 \mathrm{~mm}$. In a study on 21 patients, Dang and colleagues [30] demonstrated a statistically significant improvement in EF and PP in five and eight patients, respectively.

According to the ROBINS risk calculator, these studies are at a moderate risk of bias owing to a bias in the selection of the reported result. In fact, when analysing the primary outcome, we identified discrepancies in the measurement and statistical reporting of PC, making it difficult to draw any conclusions regarding the efficacy of IFN $\alpha-2 b$ in single-arm studies.

\subsubsection{Hyaluronic acid}

HA therapy for PD has been investigated in only two prospective studies (Table 3) [37,59]. In a study in 2000 , Lamprakopoulos and coworkers [37] administered 1500 IU HA every $2 \mathrm{wk}$, with a total of 12 injections to each palpable plaque over a period of $5 \mathrm{mo}$. The authors reported a complete resolution of PC in $32.1 \%$ of patients, resolution of plaque in $31.2 \%$ of men, and a reduction in PP in $97 \%$ of men. Statistical analysis was not reported in any of the comparisons.

In 2017, a multicentre study by Zucchi et al. [59] reported clinical outcomes of HA injections using a 10-wk cycle of weekly HA injections ( $0.8 \%$ purified sodium salt at $16 \mathrm{mg} /$ $2 \mathrm{ml})$. The authors demonstrated significant decreases in PC ( $30^{\circ}$ vs $\left.20^{\circ}\right)$ and PS (20 vs $8 \mathrm{~mm}$ ), and a slight difference in the IIEF-5 (20 vs 21) score.

\subsection{4. $\mathrm{CCH}$}

A total of eight prospective single-arm studies $[32,35,39,46,56-58]$, plus one retrospective single-arm study [25] have investigated the clinical efficacy of $\mathrm{CCH}$ for PD (Table 4). The treatment protocols exhibited considerable homogeneity between studies in terms of dosage (0.58 $\mathrm{mg}$ in all studies) and injection programmes, which ranged from three to four cycles of two injections with an interval of $\sim 24-72 \mathrm{~h}$ between each injection. Only one study evaluated the effect of $\mathrm{CCH}$ therapy combined with a vacuum device [48]. Improvements in PC ranged from $-22.6^{\circ}$ to $-12.7^{\circ}$ in studies reporting significant absolute changes [35,39,46,56-58], and -36.3\% in the one study that reported a percentage decrease [32].

Raheem and colleagues [48] evaluated the effects of $\mathrm{CCH}$ therapy combined with vacuum pump device, and reported clinically and statistically meaningful improvements in PC compared with baseline (from $54.17^{\circ}$ to $37^{\circ}$ ).

By contrast, Nguyen et al. [46] did not report any significant benefit of $\mathrm{CCH}$ injections, in patients with either early $\left(60.0^{\circ}\right.$ vs $\left.43.9^{\circ}\right)$ or chronic $\left(56.9^{\circ}\right.$ vs $\left.41.3^{\circ}\right)$ PD between baseline and the final follow-up point. Similarly, Anaissie and colleagues [25] did not demonstrate significant changes in $\mathrm{PC}$ after $\mathrm{CCH}$ injections.

Considering secondary outcomes, only Jordan [35] reported a significant reduction in plaque length $(-0.941$ inches) and width ( -0.0129 inches), whereas Raheem et al. [48] reported an increase in IIEF-5 compared with baseline (23.75 vs 20.90).

The magnitude of improvement in PP reported across studies, evaluated with the use of the Peyronie's Disease Questionnaire-penile pain domain, ranged from-5.3 to -2.4 [32,39], and a reduction of -1.64 was described when $\mathrm{CCH}$ therapy was combined with a vacuum device [48].

For PL, only the study by Levine et al. [39] reported significant changes between baseline and final follow-up $(10.6$ vs $11.0 \mathrm{~cm})$. The other three studies that reported PL did not demonstrate a significant benefit of $\mathrm{CCH}$ on this parameter $[25,46,48]$.

A study by Cocci et al. [64] found that IIEF, PC, PS, PP, and PL significantly improved after treatment and observed a mean change of $19.1^{\circ}$ of PC after treatment (three intralesional injections of $0.9 \mathrm{mg}$ performed at 4 -wk intervals).

\subsubsection{Mechanical therapy}

Four studies evaluated the efficacy of penile traction to treat PD $[33,42,43,49]$ (Table 5). Three different treatment protocols were used across studies. Gontero and colleagues [33] administered $600 \mathrm{~g}$ during the $1 \mathrm{st}$ month and $900 \mathrm{~g}$ during the 2 nd month, increasing to $1200 \mathrm{~g}$ during the 5 th and 6th months; Levine et al. [42] described a minimum of $2 \mathrm{~h}$ for up to $8 \mathrm{~h} / \mathrm{d}$ for 6 mo with the extender rods lengthened by $0.5 \mathrm{~cm}$ every $2 \mathrm{wk}$; Martínez-Salamanca and colleagues [43] used a programme of 6-9 h/d.

Gontero et al. [33] showed a slight, nonsignificant, decrease in PC from $31^{\circ}$ to $27^{\circ}$ after treatment, but improvements were not reported for the secondary outcomes.

Martínez-Salamanca et al. [43] performed a prospective nonrandomised study comparing penile traction with several types of oral therapies in the control group. This assessment has not been included in this systematic review. The authors demonstrated a decrease in PC from $33^{\circ}$ to $13^{\circ}$ after penile traction, and also reported improvements in the secondary outcomes of EF, PP, and PL after traction.

In 2008, Levine and colleagues [42] reported a $33 \%$ improvement in PC, but this outcome was not statistically significant owing to the small number of patients included in the study $(n=11)$. Moreover, $100 \%$ of patients reported some improvement in PL compared with baseline of $0.5-$ $2.5 \mathrm{~cm}$. No statistical significance was reported. 
The use of a single-arm prospective design to investigate vacuum-pump-device therapy for PD was described by Raheem et al. [49] The treatment protocol involved the use of the device for $10 \mathrm{~min}$ twice daily over a period of $12 \mathrm{wk}$. The authors reported 67\% improvement in PC from a mean at baseline of $47.6^{\circ}$, as well as improvements in PP VAS (2.2 vs 1.1$)$ and PL $(+0.5 \mathrm{~cm})$.

These data suggest that studies on penile traction and vacuum therapies are still heterogeneous to draw any conclusions, with a lack of comparative randomised studies, which could help better identify the benefit of traction in terms of functional outcomes.

\subsubsection{Other treatments}

Muñoz-Rangel and colleagues [45] investigated the role of intralesional injections of onabotulinum toxin A (100 IU) and reported significant changes in PC from $32.95^{\circ}$ to $25^{\circ}$. Moreover, the authors also reported clinical and statistically significant benefits in terms of plaque thickness $(0.34 \mathrm{vs}$ $0.27 \mathrm{~cm}$ ), IIEF-5 (16.18 vs 18.22 ) and PP VAS (from 3.36 to 1.14). A study regarding the use of thiocolchicine demonstrated significant improvements in PC, but provided only level 3 evidence using a single-arm or case-control design [22].

\section{Discussion}

In the present study, we systematically reviewed the literature investigating the use of injection therapy, vacuum devices, and penile traction therapy for the treatment of PD.

Among all retrieved articles, 12 (22.2\%) were prospective randomised studies (level of evidence 1 ) and the remaining $42(77.8 \%)$ had a case-control or a single-arm design, or were retrospective. These data provide no strong evidence that injection therapies, and traction and vacuum devices are effective for PD. Furthermore, assessment with the ROBINS calculator suggested that $51 \%$ of single-arm studies were biased in terms of measurement of outcomes and selection of reported results, which is considered a critical bias.

Regardless of these considerations, our analysis suggests a clinically meaningful effect of $\mathrm{CCH}$ and IFN $\alpha-2 b$ injection therapies in decreasing PC and increasing PL; however, only one study showed a benefit of IFN $\alpha-2 b$ in PS reduction.

For $\mathrm{CCH}$ therapy, the best support comes from two large phase III, randomised, double-blind, placebo-controlled studies (IMPRESS I and II) [6]. The results of these clinical trials showed that $\mathrm{CCH}$ can reduce PC up to $17^{\circ}$ (or $34 \%$ ), as well as improve the pain symptoms of PD and PL in some studies.

In addition to these observations, $\mathrm{CCH}$ injection was shown to slightly increase PL $(+0.4 \mathrm{~cm})$, but these findings might be attributable to the concomitant modelling procedure, rather than to a direct action of the drug itself. Indeed, penile traction therapies, such as penile modelling, have been used historically to help straighten PC in PD, using either a stretcher or a vacuum device $[33,49]$.

However, the study by Ralph and colleagues [18] that compared $\mathrm{CCH}+$ vacuum + modelling with $\mathrm{CCH}+$ vacuum did not show a significant effect on PC $\left(-23.7^{\circ}\right.$ vs $\left.-23.3^{\circ}\right)$, suggesting that the role of penile traction therapies is not limited to a specific approach. The only evidence supporting the use of traction therapies comes from three studies $[33,42,43]$, that reported clinical benefits from baseline to final follow-up; however, the data are not robust enough to drive specific recommendations.

Evidence to support clinical efficacy of verapamil and HA is weak, as few RCTs are available and data come mainly from case-control or nonrandomised clinical studies without placebo controls.

Previous research has demonstrated the efficacy of IFN- $\alpha 2 b$ injections in many in vivo and in vitro studies [51]. Two randomised, placebo-controlled trials suggest a clinical benefit of IFN- $\alpha 2$ b regarding a reduction in PC, while PS improvement was seen only in one RCT. However, improvements in PC were only $17 \%$ and $22 \%$, respectively, which certainly makes this an inferior injection therapy compared with $\mathrm{CCH}$ injections. Moreover, concerning the single-arm studies, many discrepancies in reporting measurements of $\mathrm{PC}$ and statistical analysis were found. They do not provide any valid additional information about the use of IFN- $\alpha 2$ b outside a clinical trial setting. However, in the rare case that the patient previously had a severe allergic reaction to $\mathrm{CCH}$ injections, IFN- $\alpha 2$ b can be considered as a safe, though less effective, alternative.

Level 1 evidence to support the use of calcium channel blockers comes from the RCTs by Rehman et al. [19] on verapamil and Soh et al. [21] on nicardipine. Other studies on calcium-channel blockers are either comparative (level 2 evidence) or single-arm (level 3 evidence) studies.

A study investigating different injection protocols at various doses $(10 \mathrm{mg} / 4 \mathrm{ml}, 10 \mathrm{mg} / 10 \mathrm{ml}$, and $10 \mathrm{mg} / 20 \mathrm{ml}$ ) was performed by Cavallini et al. [29], but did not reveal an advantage of any particular formulations. Verapamil injection has also been tested with EMDA, but its addition failed to demonstrate any clinical benefit [16].

Finally, studies of onabotulinum toxin A 100 IU injections [45] or thiocolchicine demonstrated significant improvements in PC, but provided only level 3 evidence in a singlearm or case-control design [22].

Despite these limitations, the current systematic review of the literature has enabled us to draw some specific recommendations on injection therapies, vacuum devices, or penile traction for PD.

\section{Conclusions}

This systematic review showed that intralesional injections of $\mathrm{CCH}$ and IFN $\alpha-2 b$ improve PC in men with PD. Moreover, $\mathrm{CCH}$ can also improve PL, and IFN $\alpha-2$ b can reduce PS. No strong recommendations can be made for other treatments, including calcium channel blockers, HA, or traction therapies. Additionally, robust RCTs should be conducted to compare injection therapies with other treatments or placebo for those treatments for which evidence is currently weak. 
Author contributions: Giorgio I. Russo had full access to all the data in the study and takes responsibility for the integrity of the data and the accuracy of the data analysis.

Study concept and design: Albersen, Russo.

Acquisition of data: Milenkovic, Russo.

Analysis and interpretation of data: Milenkovic, Russo.

Drafting of the manuscript: Milenkovic, Russo, Albersen.

Critical revision of the manuscript for important intellectual content:

Levine, Hellstrom, Ralph, Albersen.

Statistical analysis: None.

Obtaining funding: None.

Administrative, technical, or material support: None.

Supervision: Levine, Hellstrom, Ralph, Albersen.

Other: None.

Financial disclosures: Giorgio I. Russo certifies that all conflicts of interest, including specific financial interests and relationships and affiliations relevant to the subject matter or materials discussed in the manuscript (eg, employment/affiliation, grants or funding, consultancies, honoraria, stock ownership or options, expert testimony, royalties, or patents filed, received, or pending), are the following: None.

Funding/Support and role of the sponsor: None.

\section{Appendix A. Supplementary data}

Supplementary data associated with this article can be found, in the online version, at https://doi.org/10.1016/j. eururo.2018.07.005.

\section{References}

[1] Herati AS, Pastuszak AW. The genetic basis of Peyronie disease: a review. Sex Med Rev 2016;4:85-94.

[2] Hatzimouratidis K, Giuliano F, Moncada I, Muneer A, Salonia A, Verze P. EAU guidelines on erectile dysfunction, premature ejaculation, penile curvature and priapism. 2017. https://uroweb.org/ guideline/male-sexual-dysfunction/

[3] Favilla V, Russo GI, Privitera S, et al. Combination of intralesional verapamil and oral antioxidants for Peyronie's disease: a prospective, randomised controlled study. Andrologia 2014;46:936-42.

[4] Desai SS, Hentz VR. Collagenase Clostridium histolyticum for Dupuytren's contracture. Expert Opin Biol Ther 2010;10:1395-404.

[5] Carson CC, 3rd, Sadeghi-Nejad H, Tursi JP, et al. Analysis of the clinical safety of intralesional injection of collagenase Clostridium histolyticum (CCH) for adults with Peyronie's disease (PD). BJU Int 2015; 116:815-22.

[6] Gelbard M, Goldstein I, Hellstrom WJG, et al. Clinical efficacy, safety and tolerability of collagenase Clostridium histolyticum for the treatment of Peyronie disease in 2 large double-blind, randomized, placebo controlled phase 3 studies. J Urol 2013;190:199-207.

[7] Muller A, Mulhall JP. Peyronie's disease intervention trials: methodological challenges and issues. J Sex Med 2009;6:848-61.

[8] Moher D, Liberati A, Tetzlaff J, Altman DG, PRISMA Group. Preferred Reporting Items for Systematic Reviews and Meta-analyses: the PRISMA statement. PLoS Med 2009;6:e1000097.

[9] Abern MR, Larsen S, Levine LA. Combination of penile traction, intralesional verapamil, and oral therapies for Peyronie's disease. J Sex Med 2012;9:288-95.

[10] Favilla V, Russo GI, Zucchi A, et al. Evaluation of intralesional injection of hyaluronic acid compared with verapamil in Peyronie's disease: preliminary results from a prospective, double-blinded, randomized study. Andrology 2017;5:771-5.

[11] Gelbard MK, James K, Riach P, Dorey F. Collagenase versus placebo in the treatment of Peyronie's disease-a double-blind-study. J Urol 1993;149:56-8.

[12] Gennaro R, Barletta D, Paulis G. Intralesional hyaluronic acid: an innovative treatment for Peyronie's disease. Int Urol Nephrol 2015;47:1595-602.

[13] Greenfield JM, Shah SJ, Levine LA. Verapamil versus saline in electromotive drug administration for Peyronie's disease: a double-blind, placebo controlled trial. J Urol 2007;177:972-5.

[14] Hellstrom WJG, Kendirci M, Matern R, et al. Single-blind, multicenter, placebo controlled, parallel study to assess the safety and efficacy of intralesional interferon $\alpha-2 b$ for minimally invasive treatment for Peyronie's disease. J Urol 2006;176: 394-8.

[15] Kendirci M, Usta MF, Matern RV, Nowfar S, Sikka SC, Hellstrom WJ. The impact of intralesional interferon alpha-2b injection therapy on penile hemodynamics in men with Peyronie's disease. J Sex Med 2005;2:709-15.

[16] Mehrsai AR, Namdari F, Salavati A, Dehghani S, Allameh F, Pourmand G. Comparison of transdermal electromotive administration of verapamil and dexamethasone versus intra-lesional injection for Peyronie's disease. Andrology 2013;1:129-32.

[17] Montorsi F, Salonia A, Guazzoni C, et al. Transdermal electromotive multi-drug administration for Peyronie's disease: preliminary results. J Androl 2000;21:85-90.

[18] Ralph DJ, Abdel Raheem A, Liu G. Treatment of Peyronie's disease with collagenase Clostridium histolyticum and vacuum therapy: a randomized, open-label pilot study. J Sex Med 2017;14:1430-7.

[19] Rehman J, Benet A, Melman A. Use of intralesional verapamil to dissolve Peyronie's disease plaque: a long-term single-blind study. Urology 1998;51:620-6.

[20] Shirazi M, Haghpanah AR, Badiee M, Afrasiabi MA, Haghpanah S. Effect of intralesional verapamil for treatment of Peyronie's disease: a randomized single-blind, placebo-controlled study. Int Urol Nephrol 2009;41:467-71.

[21] Soh J, Kawauchi A, Kanemitsu N, et al. Nicardipine vs. saline injection as treatment for Peyronie's disease: a prospective, randomized, single-blind trial. J Sex Med 2010;7:3743-9.

[22] Toscano Jr L, Rezende MV, Mello LF, Paulillo D, Glina S. A prospective, randomized, single-blind study comparing intraplaque injection of thiocolchicine and verapamil in Peyronie's disease: a pilot study. Int Braz J Urol 2016;42:1005-9.

[23] Yafi FA, Pinsky MR, Stewart C, et al. The effect of duration of penile traction therapy in patients undergoing intralesional injection therapy for Peyronie's disease. J Urol 2015;194:754-8.

[24] Ahuja S, Bivalacqua TJ, Case J, Vincent M, Sikka SC, Hellstrom WJG. A pilot study demonstrating clinical benefit from intralesional interferon alpha $2 \mathrm{~B}$ in the treatment of Peyronie's disease. J Androl 1999;20:444-8.

[25] Anaissie J, Yafi FA, DeLay KJ, Traore EJ, Sikka SC, Hellstrom WJG. Impact of number of cycles of collagenase Clostridium histolyticum on outcomes in patients with Peyronie's disease. Urology 2017;100:125-30.

[26] Astorga R, Cantero O, Contreras D, et al. Intralesional recombinant interferon alpha-2b in Peyronie's disease. Arch Esp Urol 2000;53: 665-71.

[27] Bennett NE, Guhring P, Mulhall JP. Intralesional verapamil prevents the progression of Peyronie's disease. Urology 2007;69:1181-4.

[28] Brake M, Loertzer H, Horsch R, Keller H. Treatment of Peyronie's disease with local interferon- $\alpha$ 2b. BJU Int 2001;87:654-7.

[29] Cavallini G, Modenini F, Vital G. Open preliminary randomized prospective clinical trial of efficacy and safety of three different 
verapamil dilutions for intraplaque therapy of Peyronie's disease. Urology 2007;69:950-4.

[30] Dang G, Matern R, Bivalacqua TJ, Sikka S, Hellstrom WJG. Intralesional interferon- $\alpha-2 b$ injections for the treatment of Peyronie's disease. Southern Med J 2004;97:42-6.

[31] Dell'Atti L. Tadalafil once daily and intralesional verapamil injection: a new therapeutic direction in Peyronie's disease. Urol Ann 2015;7:345-9.

[32] Goldstein I, Knoll LD, Lipshultz LI, Smith T, Kaufman GJ, McMahon CG. Changes in the effects of Peyronie's disease after treatment with collagenase Clostridium histolyticum: male patients and their female partners. Sex Med 2017;5:E124-30.

[33] Gontero P, Di Marco M, Giubilei G, et al. Use of penile extender device in the treatment of penile curvature as a result of Peyronie's disease. Results of a phase II prospective study. J Sex Med 2009;6:558-66.

[34] Heidari M, Nejadi JR, Ghate A, Delfan B, Iran-Pour E. Evaluation of Intralesional injection of Verapamil in treatment of Peyronie's disease. J Pak Med Assoc 2010;60:291-3.

[35] Jordan GH. The use of intralesional clostridial collagenase injection therapy for Peyronie's disease: a prospective, single-center, nonplacebo-controlled study. J Sex Med 2008;5:180-7.

[36] Judge IS, Wisniewski ZS. Intralesional interferon in the treatment of Peyronie's disease: a pilot study. Br J Urol 1997;79:40-2.

[37] Lamprakopoulos A, Zorzos I, Lykourinas I. The use of betamethasone and hyaluronidase injections in the treatment of Peyronie's disease. Scand J Urol Nephrol 2000;34:355-60.

[38] Levine LA. Treatment of Peyronie's disease with intralesional verapamil injection. J Urol 1997;158:1395-9.

[39] Levine LA, Cuzin B, Mark S, et al. Clinical safety and effectiveness of collagenase Clostridium histolyticum injection in patients with Peyronie's disease: a phase 3 open-label study. J Sex Med 2015;12:248-58.

[40] Levine LA, Goldman KE, Greenfield JM. Experience with intraplaque injection of verapamil for Peyronie's disease. J Urol 2002;168:6215, discussion 625-6.

[41] Levine LA, Merrick PF, Lee RC. Intralesional verapamil injection for the treatment of Peyronie's disease. J Urol 1994;151:1522-4.

[42] Levine LA, Newell M, Taylor FL. Penile traction therapy for treatment of Peyronie's disease: a single-center pilot study. J Sex Med 2008;5:1468-73.

[43] Martínez-Salamanca JI, Egui A, Moncada I, et al. Acute phase Peyronie's disease management with traction device: a nonrandomized prospective controlled trial with ultrasound correlation. J Sex Med 2014;11:506-15.

[44] Moskovic DJ, Alex B, Choi JM, Nelson CJ, Mulhall JP. Defining predictors of response to intralesional verapamil injection therapy for Peyronie's disease. BJU Int 2011;108:1485-9.

[45] Muñoz-Rangel CA, Fernandez-Vivar E, Banuelos-Gallo RA, et al. Minimally invasive therapy using intralesional onabotulinumtoxinA in Peyronie's disease. Urol J 2015;12:2105-10.

[46] Nguyen HMT, Anaissie J, DeLay KJ, Yafi FA, Sikka SC, Hellstrom WJG. Safety and efficacy of collagenase Clostridium histolyticum in the treatment of acute-phase Peyronie's disease. J Sex Med 2017; 14:1220-5.

[47] Polat O, Gul O, Ozbey I, Ozdikici M, Bayraktar Y. Peyronie's disease: intralesional treatment with interferon alpha-2A and evaluation of the results by magnetic resonance imaging. Int Urol Nephrol 1997;29:465-71.

[48] Raheem AA, Capece M, Kalejaiye O, et al. Safety and effectiveness of collagenase clostridium histolyticum in the treatment of Peyronie's disease using a new modified shortened protocol. BJU Int 2017;120:717-23.

[49] Raheem AA, Garaffa G, Raheem TA, et al. The role of vacuum pump therapy to mechanically straighten the penis in Peyronie's disease. BJU Int 2010;106:1178-80.

[50] Riedl CR, Plas E, Engelhardt P, Daha K, Pfluger H. Iontophoresis for treatment of Peyronie's disease. J Urol 2000;163:95-9.

[51] Stewart CA, Yafi FA, Knoedler M, et al. Intralesional injection of interferon-alpha2b improves penile curvature in men with Peyronie's disease independent of plaque location. J Urol 2015;194:1704-7.

[52] Tuygun C, Ozok UH, Gucuk A, Bozkurt IH, Imamoglu MA. The effectiveness of transdermal electromotive administration with verapamil and dexamethasone in the treatment of Peyronie's disease. Int Urol Nephrol 2009;41:113-8.

[53] Wegner HEH, Andresen R, Knipsel HH, Miller K. Treatment of Peyronie's-disease with local interferon-alpha-2b. Eur Urol 1995;28:236-40.

[54] Wegner HEH, Andresen R, Knispel HH, Miller K. Local interferonalpha2b is not an effective treatment in early-stage Peyronie's disease. Eur Urol 1997;32:190-3.

[55] Wolff B, Peyronnet B, Cattarino S, et al. Intralesional injections for early Peyronie disease: standardized assessment and analysis of predictive factors for treatment response. Urology 2015;86: 57-61.

[56] Yang KK, Bennett N. Peyronie's disease and injectable collagenase Clostridium histolyticum: safety, efficacy, and improvements in subjective symptoms. Urology 2016;94:143-7.

[57] Ziegelmann MJ, Viers BR, McAlvany KL, Bailey GC, Savage JB, Trost LW. Restoration of penile function and patient satisfaction with intralesional collagenase Clostridium histolyticum injection for Peyronie's disease. J Urol 2016;195:1051-6.

[58] Ziegelmann MJ, Viers BR, Montgomery BD, Westerman ME, Savage JB, Trost LW. Self-reported clinical meaningfulness early in the treatment course predicts objective outcomes in men undergoing collagenase Clostridium histolyticum injections for Peyronie disease. Urology 2017;106:107-12.

[59] Zucchi A, Costantini E, Cai T, et al. Intralesional injection of hyaluronic acid in patients affected with Peyronie's disease: preliminary results from a prospective, multicenter, pilot study. Sex Med 2016;4:E85-90.

[60] Higgins JP, Altman DG, Gotzsche PC, et al. The Cochrane Collaboration's tool for assessing risk of bias in randomised trials. BMJ 2011;343:d5928.

[61] Sterne JA, Hernan MA, Reeves BC, et al. ROBINS-I: a tool for assessing risk of bias in non-randomised studies of interventions. BMJ 2016;355:i4919.

[62] Ziegelmann MJ, Viers BR, Montgomery BD, Avant RA, Savage JB, Trost LW. Clinical experience with penile traction therapy among men undergoing collagenase Clostridium histolyticum for Peyronie disease. Urology 2017;104:102-9.

[63] Inal T, Tokatli Z, Akand M, Ozdiler E, Yaman O. Effect of intralesional interferon-alpha $2 \mathrm{~b}$ combined with oral vitamin $\mathrm{E}$ for treatment of early stage Peyronie's disease: a randomized and prospective study. Urology 2006;67:1038-42.

[64] Cocci A, Russo GI, Salonia A, et al. Predictive factors of patients' and their partners' sexual function improvement after collagenase Clostridium histolyticum injection for Peyronie's disease: results from a multi-center single-arm study. J Sex Med 2018;15:716-21. 